\title{
STABILITY OF THE LOBACEVSKI EQUATION
}

\author{
GWANG HUI KIM
}

Dedicated to Themistocles M. Rassias on the occasion of his sixtieth birthday

Abstract. The aim of this paper is to investigate the superstability of the Lobacevski equation

$$
f\left(\frac{x+y}{2}\right)^{2}=f(x) f(y)
$$

which is bounded by the unknown functions $\varphi(x)$ or $\varphi(y)$. The obtained result is a generalization of P. Găvruta's result in 1994 .

\section{INTRODUCTION}

The stability problem of the functional equation was conjectured by Ulam [28] during the conference in the university of Wisconsin in 1940. In the next year, it was solved by Hyers [12] in the case of additive mapping, which is called the Hyers-Ulam stability. Thereafter, this problem was improved by D.G. Bourgin [6], T. Aoki [1], Th.M. Rassias [22], R. Ger [11], P. Găvruta [9], and G. L. Forti [8], in which Rassias's result is called the Hyers-Ulam-Rassias stability.

Thereafter some researchers in his named stability and its generalization have studied the papers $([14]-[21])$.

In 1979, J. Baker et al. in [5] developed the superstability, which is if $f$ satisfies the stability inequality $\left|E_{1}(f)-E_{2}(f)\right| \leq \varepsilon$, then either $f$ is bounded or $E_{1}(f)=$ $E_{2}(f)$.

Baker [4] showed the superstability of the cosine functional equation (also called the d'Alembert functional equation)

$$
f(x+y)+f(x-y)=2 f(x) f(y) .
$$

Date: Received: October 10, 2010; Revised: November 16, 2010.

(c) 2010 N.A.G.

2000 Mathematics Subject Classification. Primary 39B82, 39B52.

Key words and phrases. Hyers-Ulam-Rassias stability; superstability; Lobacevski equation; d'Alembert functional equation; sine functional equation. 
The stability of the generalized functional equations of $(\mathrm{A})$

$$
\begin{aligned}
& f(x+y)+f(x-y)=2 f(x) g(y), \\
& f(x+y)-f(x-y)=2 g(x) f(y),
\end{aligned}
$$

was researched in papers $([3,13,15])$, in which $\left(A_{f g}\right)$, as the generalization of the cosine functional equation (A), is called the Wilson functional equation, and $\left(\overline{T_{g f}}\right)$ is the mixed trigonometric functional equation.

The superstability bounded by a constant for the sine functional equation

$$
f\left(\frac{x+y}{2}\right)^{2}-f\left(\frac{x-y}{2}\right)^{2}=f(x) f(y),
$$

was investigated by P.W. Cholewa [7] and was improved by R. Badora and R. Ger [3].

Găvruta [10] proved the superstability of the Lobacevski equation

$$
f\left(\frac{x+y}{2}\right)^{2}=f(x) f(y)
$$

under the condition bounded by a constant, which is similar to the sine functional equation and the exponential functional equation.

The aim of this paper is a generalization of P. Găvruta's result [10], i.e. which is to investigate the superstability of the Lobacevski equation (L) under the condition bounded by a function (i.e. sense of Găvruta). Furthermore, the range of the function in all results expands to the Banach space. For an simple example of this equation, we can find the functional equation

$$
\left(e^{x+y}\right)^{2}=e^{x} e^{y} .
$$

In this paper, let $(G,+)$ be a uniquely 2-divisible Abelian group (i.e. for each $x \in G$ there exists a unique $y \in G$ such that $y+y=x$ : such a $y$ will be denoted by $\frac{x}{2}$ ), $\mathbb{C}$ the field of complex numbers, and $\mathbb{R}$ the field of real numbers, $\mathbb{R}_{+}$the set of positive reals. We assume that $f$ and $g$ are nonzero functions, $\varepsilon$ is a nonnegative real constant, and $\varphi: G \rightarrow \mathbb{R}_{+}$be a mapping.

\section{Stability of the functional Equation (L)}

We will investigate the superstability of the Lobacevski equation (L).

Theorem 1. Let $\varphi$ be a continuous function such that $\lim _{n \rightarrow \infty} \varphi\left(x_{n}\right)<\infty$. Suppose that $f: G \rightarrow \mathbb{C}$ satisfies the inequality

$$
\left|f\left(\frac{x+y}{2}\right)^{2}-f(x) f(y)\right| \leq \varphi(y)
$$

for all $x, y \in G$.

Then either $|f(x)| \leq \frac{|f(0)|+\sqrt{|f(0)|^{2}+4 \varphi(0)}}{2}$ for all $x \in G$ or $f$ satisfies (L). 
Proof. Suppose that there exists $x_{0} \in G$ such that

$$
\left|f\left(x_{0}\right)\right|>\frac{|f(0)|+\sqrt{|f(0)|^{2}+4 \varphi(0)}}{2} .
$$

We prove that there exists a sequence $\left\{x_{n}\right\}$ in $G$ such that

$$
\lim _{n \rightarrow \infty}\left|f\left(x_{n}\right)\right|=\infty \text {. }
$$

Taking $y=0$ in (2.1) and replacing $x$ by $2 x$, we obtain

$$
\left|f(x)^{2}-f(0) f(2 x)\right| \leq \varphi(0)
$$

If $f(0)=0$, from (2.4) it follows that

$$
|f(x)| \leq \sqrt{\varphi(0)}, \quad \forall x \in G .
$$

and from $(2.2)$ we obtain $\left|f\left(x_{0}\right)\right|>\sqrt{\varphi(0)}$, contradiction.

Thus $f(0) \neq 0$. From (2.4) we also obtain

$$
\begin{aligned}
|f(0)||f(2 x)| & =\left|f(x)^{2}+\left(f(0) f(2 x)-f(x)^{2}\right)\right| \\
& \geq|f(x)|^{2}-\left|f(0) f(2 x)-f(x)^{2}\right| \\
& \geq|f(x)|^{2}-\varphi(0), \quad \forall x \in G .
\end{aligned}
$$

Thus

We denote

$$
|f(2 x)| \geq \frac{|f(x)|^{2}-\varphi(0)}{|f(0)|}, \quad \forall x \in G .
$$

$$
\alpha=\frac{|f(0)|+\sqrt{|f(0)|^{2}+4 \varphi(0)}}{2}, \quad \beta=\left|f\left(x_{0}\right)\right|-\alpha .
$$

Then

$$
\left|f\left(x_{0}\right)\right|=\alpha+\beta
$$

and by (2.2), $\beta>0$.

We will prove by induction that

$$
f\left(2^{n} x_{0}\right) \geq \alpha+2^{n} \beta, \quad \forall n \in N .
$$

Taking $x=x_{0}$ in (2.6) and making use of (2.8) we obtain

$$
\left|f\left(2 x_{0}\right)\right| \geq \frac{(\alpha+\beta)^{2}-\varphi(0)}{|f(0)|}=\alpha+\frac{2 \alpha \beta+\beta^{2}}{|f(0)|} \geq \alpha+2 \beta
$$

because

and

$$
\alpha^{2}=\alpha|f(0)|+\varphi(0)
$$

$$
\alpha \geq|f(0)|, \quad \beta>0 .
$$

Thus (2.9) is valid for $n=1$.

Now suppose that (2.9) is true for a $n \in N$. Taking $x=2^{n} x_{0}$ in (2.6) and applying (2.11) we get

$$
f\left(2^{n+1} x_{0}\right) \geq \frac{\left(\alpha+2^{n} \beta\right)^{2}-\varphi(0)}{|f(0)|}=\alpha+\frac{2^{n+1} \alpha \beta+2^{2 n} \beta^{2}}{|f(0)|} \geq \alpha+2^{n+1} \beta,
$$


which completes the induction.

In virtue of (2.11) and(2.12) the sequence $x_{n}=2^{n} x_{0}, n \in N$ satisfies (2.3).

We consider $x, y \in G$. From (2.1) it follows that

$$
\left|f\left(\frac{x_{n}+x}{2}\right)^{2}-f\left(x_{n}\right) f(x)\right| \leq \varphi(x) \quad \forall n \in N
$$

i.e.

$$
\left|f(x)-\frac{f\left(\frac{x_{n}+x}{2}\right)^{2}}{f\left(x_{n}\right)}\right| \leq \frac{\varphi(x)}{\left|f\left(x_{n}\right)\right|} \quad \forall n \in N
$$

whence by (2.3) we obtain

$$
f(x)=\lim _{n \rightarrow \infty} \frac{f\left(\frac{x+x_{n}}{2}\right)^{2}}{f\left(x_{n}\right)} .
$$

From (2.15) we have

$$
f(x) f(y)=\left[\lim _{n \rightarrow \infty} \frac{f\left(\frac{x+x_{n}}{2}\right) f\left(\frac{y+x_{n}}{2}\right)}{f\left(x_{n}\right)}\right]^{2}
$$

and

$$
f\left(\frac{x+y}{2}\right)^{2}=\left[\lim _{n \rightarrow \infty} \frac{f\left(\frac{x+y+2 x_{n}}{4}\right)^{2}}{f\left(x_{n}\right)}\right]^{2}
$$

The relation (2.1) implies

$$
\left|\frac{f\left(\frac{x+x_{n}}{2}\right) f\left(\frac{y+x_{n}}{2}\right)}{f\left(x_{n}\right)}-\frac{f\left(\frac{x+y+2 x_{n}}{4}\right)^{2}}{f\left(x_{n}\right)}\right| \leq \frac{\varphi\left(\frac{y+x_{n}}{2}\right)}{\left|f\left(x_{n}\right)\right|}, \quad \forall n \in N .
$$

Using by the assumption of $\varphi$ and (2.3), the right side of (2.18) converges to zero as $n \rightarrow \infty$. From (2.16), (2.17), and (2.18), we arrived the required result (L).

Remark 1. If $f: G \rightarrow \mathbb{R}$ is bounded function satisfying $(\mathrm{L})$, that is

$$
\left|f\left(\frac{x+y}{2}\right)^{2}-f(x) f(y)\right| \leq \varphi(y)
$$

then

$$
|f(x)| \leq \frac{|f(0)|+\sqrt{|f(0)|^{2}+4 \varphi(0)}}{2} \text { for all } x \in G .
$$

In fact, suppose that $f: G \rightarrow \mathbb{R}$ satisfies (2.19) and

$$
M:=\sup \{|f(x)|: x \in G\}>\frac{|f(0)|+\sqrt{|f(0)|^{2}+4 \varphi(0)}}{2} .
$$

There exists a sequence $\left\{\left(x_{n}\right): n \in \mathbb{N}\right\}$ in $G$ such that

$$
\lim _{n \rightarrow \infty}\left|f\left(\frac{x_{n}}{2}\right)\right|=M \text {. }
$$


Then for sufficiently large $n \in \mathbb{N}$, we have

$$
\begin{aligned}
\mid f\left(\frac{x_{n}+0}{2}\right)^{2} & -f\left(x_{n}\right) f(0) \mid \\
& \geq\left.|| f\left(\frac{x_{n}+0}{2}\right)\right|^{2}-\left|f\left(x_{n}\right)\right||f(0)| \mid \\
& \geq M^{2}-M|f(0)| .
\end{aligned}
$$

Moreover from (2.21) and (2.22), we have

$$
\lim _{n \rightarrow \infty}\left|f\left(\frac{x_{n}+0}{2}\right)^{2}-f\left(x_{n}\right) f(0)\right| \geq M^{2}-M|f(0)|>\varphi(0) .
$$

We know from (2.19) that

$$
\left|f\left(\frac{x_{n}+0}{2}\right)^{2}-f\left(x_{n}\right) f(0)\right| \leq \varphi(0)
$$

for some $n \in \mathbb{N}$, which contradicts (2.23).

Theorem 2. Let $\varphi$ be a continuous function such that $\lim _{n \rightarrow \infty} \varphi\left(x_{n}\right)<\infty$. Suppose that $f: G \rightarrow \mathbb{C}$ satisfies the inequality

$$
\left|f\left(\frac{x+y}{2}\right)^{2}-f(x) f(y)\right| \leq \varphi(x)
$$

for all $x, y \in G$.

Then either $|f(x)| \leq \frac{|f(0)|+\sqrt{|f(0)|^{2}+4 \varphi(0)}}{2}$ for all $x \in G$ or $f$ satisfies (L).

Proof. The proof is very similar to Theorem 1.

Corollary 1. Let $\varphi$ be a continuous function such that $\lim _{n \rightarrow \infty} \varphi\left(x_{n}\right)<\infty$. Suppose that $f: G \rightarrow \mathbb{C}$ satisfies the inequality

$$
\left|f\left(\frac{x+y}{2}\right)^{2}-f(x) f(y)\right| \leq \min \{\varphi(x), \varphi(y)\}
$$

for all $x, y \in G$.

Then either $|f(x)| \leq \frac{|f(0)|+\sqrt{|f(0)|^{2}+4 \varphi(0)}}{2}$ for all $x \in G$ or $f$ satisfies (L).

Corollary 2. Suppose that $f: G \rightarrow \mathbb{C}$ satisfies the inequality

$$
\left|f\left(\frac{x+y}{2}\right)^{2}-f(x) f(y)\right| \leq \varepsilon
$$

for all $x, y \in G$.

Then either $|f(x)| \leq \frac{|f(0)|+\sqrt{|f(0)|^{2}+4 \varepsilon}}{2}$ for all $x \in G$ or $f$ satisfies $(\overline{\mathrm{L}})$.

In all the above results presented in this paper, the range of functions on the Abelian group can be extended to the semisimple commutative Banach space. 
Theorem 3. Let $(E,\|\cdot\|)$ be a semisimple commutative Banach space. Let $\varphi$ be a continuous function such that $\lim _{n \rightarrow \infty} \varphi\left(x_{n}\right)<\infty$.

For an arbitrary linear multiplicative functional $x^{*} \in E^{*}$,

Assume that $f: G \rightarrow E$ satisfies one of each inequalities

$$
\left\|f\left(\frac{x+y}{2}\right)^{2}-f(x) f(y)\right\| \leq \begin{cases}(i) & \varphi(y) \\ (i i) & \varphi(x)\end{cases}
$$

for all $x, y \in G$

Then either $\left\|\left(x^{*} \circ f\right)(x)\right\| \leq \frac{|f(0)|+\sqrt{|f(0)|^{2}+4 \varphi(0)}}{2}$ for all $x \in G$ or $f$ satisfies (L).

Proof. For the case $\varphi(y)$, assume that (2.26) holds and arbitrarily fixes a linear multiplicative functional $x^{*} \in E^{*}$. As is well known, we have $\left\|x^{*}\right\|=1$, hence, for every $x, y \in G$, we have

$$
\begin{aligned}
\varphi(x) & \geq\left\|f\left(\frac{x+y}{2}\right)^{2}-f(x) f(y)\right\| \\
& =\sup _{\left\|y^{*}\right\|=1}\left|y^{*}\left(f\left(\frac{x+y}{2}\right)^{2}-f(x) f(y)\right)\right| \\
& \geq\left|x^{*}\left(f\left(\frac{x+y}{2}\right)^{2}\right)-x^{*}(f(x)) x^{*}(f(y))\right|,
\end{aligned}
$$

which states that the superposition $x^{*} \circ f$ yield a solution of inequality (2.1) in Theorem 1. Since, by assumption, the boundedness of the superposition $x^{*} \circ f$, an appeal to Theorem 1 shows that the superposition $x^{*} \circ f$ solves $(\mathrm{L})$, that is

$$
\left(x^{*} \circ f\right)\left(\frac{x+y}{2}\right)^{2}=\left(x^{*} \circ f\right)(x)\left(x^{*} \circ f\right)(y) .
$$

Since $x^{*}$ is a linear multiplicative functional, we get

$$
x^{*}\left(f\left(\frac{x+y}{2}\right)^{2}-f(x) f(y)\right)=0 .
$$

Hence a unrestricted choice of $x^{*}$ implies that

$$
f\left(\frac{x+y}{2}\right)^{2}-f(x) f(y) \in \bigcap\left\{\operatorname{ker} x^{*}: x^{*} \in E^{*}\right\}
$$

Since the space $E$ is semisimple, $\bigcap\left\{\operatorname{ker} x^{*}: x^{*} \in E^{*}\right\}=0$, which means that $f$ satisfies the claimed equation (L).

The case $\varphi(x)$ also runs along the above process.

Corollary 3. Let $(E,\|\cdot\|)$ be a semisimple commutative Banach space. Let $\varphi$ be a continuous function such that $\lim _{n \rightarrow \infty} \varphi\left(x_{n}\right)<\infty$.

For an arbitrary linear multiplicative functional $x^{*} \in E^{*}$,

Assume that $f: G \rightarrow E$ satisfies one of each inequalities

$$
\left\|f\left(\frac{x+y}{2}\right)^{2}-f(x) f(y)\right\| \leq \varepsilon
$$


for all $x, y \in G$

Then either $\left\|\left(x^{*} \circ f\right)(x)\right\| \leq \frac{|f(0)|+\sqrt{|f(0)|^{2}+4 \varphi(0)}}{2}$ for all $x \in G$ or $f$ satisfies (L).

\section{REFERENCES}

[1] T. Aoki, On the stability of the linear transformation in Banach spaces, J. Math. Soc. Japen, 2 (1950), 64-66.

[2] R. Badora, On the stability of cosine functional equation, Rocznik Naukowo-Dydak., Prace Mat., 15 (1998), 1-14.

[3] R. Badora and R. Ger, On some trigonometric functional inequalities, Functional Equations- Results and Advances, (2002), 3-15.

[4] J. A. Baker, The stability of the cosine equation, Proc. Amer. Math. Soc., 80 (1980), 411-416.

[5] J. Baker, J. Lawrence and F. Zorzitto, The stability of the equation $f(x+y)=f(x) f(y)$, Proc. Amer. Math. Soc., 74 (1979), 242-246.

[6] D. G. Bourgin, Multiplicative transformations, Proc. Nat. Academy Sci. of U.S.A., 36 (1950) 564-570.

[7] P.W. Cholewa, The stability of the sine equation, Proc. Amer. Math. Soc., 88 (1983). 631-634.

[8] G. L. Forti, Hyers-Ulam stability of functional equations in several variables, Aequationes Mathematicae

[9] P. Găvruta, A Generalization of the Hyers-Ulam-Rassias Stability of Approximately Additive Mapping, Journal of Mathematical Analysis and Applications 184, 184 (1994), 431-436.

[10] P. Găvruta, On the stability of some functional equation, Stability of Mappings of HyersUlam Type, Hardronic Press, (1994), 93-98.

[11] R. Ger, Superstability is not natural,Roczik Naukowo-Dydaktyczny WSP w Krakowie, Prace Mat., 159 (1993) 109-123.

[12] D. H. Hyers, On the stability of the linear functional equation, Proc. Nat. Acad. Sci. USA., 27 (1941), 222-224.

[13] Pl. Kannappan and G. H. Kim, On the stability of the generalized cosine functional equations, Ann. Acad. Paedagog. Crac. Stud. Math., 1 (2001), 49-58.

[14] G. H. Kim, A generalization of Hyers-Ulam-Rassias Stability of the G-Functional Equation, Math. Ineq. \& Appl., 10, No. 2 (2007), 351-58.

[15] Anal \& Appl., 325 (2007), 237-248.

[16] , A Stability of the generalized sine functional equations, Jour. Math. Anal \& Appl., 331 (2007), 886-894.

[17] , K. W. Jun and Y. H. Lee, On The Hyers-Ulam-Rassias Stability of a Pexiderizes Mixes Type Quadratic functional equation, J. Chungcheong Math. Soc., 20, No. 2 (2007), 117-137.

[18] _ On the Hyers-Ulam-Rassias stability of functional equations in n-variables, Jour. Math. Anal \& Appl., 299, Issue 2 (2004), 375-391.

[19] _ A generlization of the Hyers- Ulam-Rassia Stability of the beta functional equation, Publ. Mathematics, 59 (2001), 111-119.

[20] _ Y. W. Lee and K. S. Ji, Modified Hyers-Ulam-Rassias Stability of functional equations with square-symmetric Operation, Comm. Korean Math.Soc., 16, No. 2 (2001), $211-223$.

[21] $f\left(x^{2}\right)-f\left(y^{2}\right)+r f(x y)$, J. Chungcheong Math. Soc., 10 (1997), 109-116.

[22] Th. M. Rassias, On the stability of the linear mapping in Banach spaces, Proc. Amer. Math. Soc. 72(1978), 297-300.

[23] _ On a Modified Hyers-Ulam Sequence, Jour. Math. Anal \& Appl. 158, 158 (1991), 106-113. 
[24] _ and P. Semrl, On the Behavior of Mappings Which Do Not Satisfy Hyers-Ulam Stability, Proc. Amer. Math. Soc., 114, No. 4 (1992), 989-993.

[25] _ On the Hyers-Ulam Stability of Linear Mappings, Jour. Math. Anal \& Appl., 173, No. 2 (1993), 325-338.

[26] _ On the Stability of functional equations in Banach Spaces, Jour. Math. Anal \& Appl., 251 (2000), 264-284.

[27] , The Problem of S. M. Ulam for Approximately Multiplicative Mappings, Jour. Math. Anal \& Appl., 246 (2000), 352-378.

[28] S. M. Ulam, "Problems in Modern Mathematics" Chap. VI, Science editions, Wiley, New York, (1964)

Department of Mathematics, Kangnam University, Yongin, Gyeonggi 446-702, REPUBlic OF Korea

E-mail address: ghkim@kangnam.ac.kr 